\title{
Estudio contrastivo y multimodal inglés- francés de la traducción de construcciones expresivas de alivio en cómics
}

\section{English-French contrastive and multimodal study of the translation of expressive relief constructions in comics}

\author{
Fernando Casanova Martínez \\ Universidad de Murcia \\ fernando.casanova1@um.es
}

\begin{abstract}
Translating interjections, onomatopoeias and inarticulate sounds has become a challenge due to the lack of boundaries of each unit and their relation to emotions. The main objective of this article is the contrastive and multimodal analysis of English expressive relief constructions in their passage into French. For this purpose, a corpus constituted by series of American comics is established, which has allowed us to analyze the emotion of relief and the properties of each construction from a verbal and visual perspective, highlighting its semantic, phonetic, pragmatic, morphosyntactic, and paralinguistic aspect. Although relief is an emotion present in our everyday life, comics do not prolifically represent that reality in their constitution. However, of the relief constructions found, most are modified into French, despite the tendency to prevail the English original. Thus, this study provides methodological keys to translate the expressive constructions of relief.
\end{abstract}

\section{Key-words}

expressive constructions, relief, multimodality, translation.

\begin{abstract}
Résumé
La traduction des interjections, des onomatopées et des sons inarticulés est devenue un défi en raison du manque de délimitation de chaque unité et de leur relation avec les émotions. L'objectif principal est l'analyse contrastive et multimodale des constructions expressives de soulagement anglais dans leur passage en français. Dans ce but, un corpus constitué par des séries de comics américains est établi afin d'analyser l'émotion de soulagement et les propriétés verbales et visuelles de chaque construction, en soulignant ses aspects sémantiques, phonétiques, pragmatiques, morphosyntaxiques et paralinguistiques. Bien que le soulagement soit une émotion présente dans notre vie quotidienne, la bande dessinée ne représente pas de manière prolifique cette réalité. Cependant, la plupart des constructions de soulagement trouvées sont modifiées en français, malgré la tendance à faire prévaloir l'original anglais. Ainsi, l'approche multimodale est essentielle à ces constructions et à leur rapport au soulagement.
\end{abstract}

Mots-clés

constructions expressives, soulagement, multimodalité, traduction. 


\section{Introducción}

Hallar una definición o descripción funcional para los elementos lingüísticos comúnmente denominados como interjecciones se ha convertido en un problema en el seno de los estudios lingüísticos debido a los límites difusos entre conceptos como onomatopeya, interjección y sonido inarticulado (Almela Pérez, 1982; López Bobo, 2002). Una cualidad que diferencia a estas unidades radica en su fuerza ilocutiva: las interjecciones sí que tienen fuerza ilocutiva, esto es, pueden manifestar una intención comunicativa concreta, mientras que las onomatopeyas no. Por su parte, los sonidos inarticulados se consideran como señales sintomáticas (Alonso Cortés, 1999).

No obstante, este problema para establecer límites entre estas unidades expresivas no solo recae sobre los estudios referidos al español, sino que en francés sucede el mismo conflicto:

Bien que les onomatopées diffèrent des interjections en ce sens qu'elles ne dénotent pas d'émotions ni de sensations, elles partagent avec elles des propriétés phonétiques et phonologiques, ainsi que leur nature lexicale. En plus, elles ont une portée pragmatique à l'oral ou à l'écrit, son utilisation étant guidée pour créer un effet quelconque ${ }^{1}$. (Klett, 2020: 16)

El problema es que tanto las onomatopeyas como las interjecciones se refieren a sonidos humanos y la propuesta que sugiere la autora es la formación de la siguiente tríada: interjecciones, onomatopeyas y pseudogritos, siendo estos últimos tomados de Bottineau (2013/4). Tanto onomatopeyas como pseudogritos estarían codificados sobre la idea de la reproducción mimética de sonidos, pero, mientras que las onomatopeyas reproducen sonidos de animales, de la naturaleza o de objetos (pan!, ipum!) $)^{2}$, los pseudogritos reflejan emociones heterogéneas de origen humano que el hablante debe interiorizar, puesto que son propias de cada cultura (aïe!, ;ay!). Y, por su parte, las interjecciones quedarían fuera de ese fenómeno mimético (miam!, ; inam!).

Comparando esta literatura referente a las dos lenguas, se observa que o bien la fuerza ilocutiva o la relación mimética se toman como puntos de referencia para distinguir a las interjecciones y a las onomatopeyas, mientras que los sonidos inarticulados y los pseudogritos son similares en cuanto a su origen mimético y a su función como indicios emotivos o sensitivos. Sin embargo, pese a estas clasificaciones encaminadas al discernimiento de los términos, estos permanecen siendo confusos en ambos idiomas.

Aunque esta cuestión es mucho más amplia y debatida en numerosas investigaciones

1 Traducción: "Aunque las onomatopeyas se diferencian de las interjecciones en que no denotan emociones o sensaciones, comparten con ellas propiedades fonéticas y fonológicas, así como su naturaleza léxica. Además, tienen un alcance pragmático en el habla o la escritura con la finalidad de crear algún efecto".

2 De este par de ejemplos, el primero en francés se corresponde con el que aporta Bottineau (2013/4: 99) y el segundo es la adaptación del término al español. 
lingüísticas, no es posible abordar toda esta problemática, dado que son cuantiosas las consideraciones al respecto que culminan en una falta de consenso para su delimitación. Por esta razón, en este artículo se sugiere el término amplio de construcciones expresivas, siguiendo a la Lingüística Cognitiva y más concretamente a la Gramática de Construcciones (Goldberg, 1995). Las construcciones expresivas (CCEE a partir de ahora) son aquellos elementos lingüísticos cargados de un uso expresivo-emocional en un determinado contexto, que englobarían a las interjecciones, onomatopeyas y sonidos inarticulados. Esta definición es muy similar a la que proporcionan Ellis et al. (2016) sobre las interjecciones a las consideran como símbolos lingüísticos cargados de significado.

A continuación, se precisan qué características asociadas tradicionalmente a la categoría de la interjección se toman como base en este trabajo y se incorporan al concepto de construcción expresiva referido más arriba: las interjecciones son unidades comunicativas con una sintaxis anómala, pero que semántica y entonativamente son completas (Cuenca, 2000: 29). Asimismo, presentan una naturaleza pragmática cuya interpretación depende fuertemente del contexto inmediato del hablante, pudiendo proyectar una emoción, un sentimiento o una actitud (Goddard, 2013: 54). En este sentido, Goddard, siguiendo la terminología de Wilkins (1992), las denomina como deícticos complejos porque pueden conllevar a la construcción por parte de los oyentes/lectores de explicaturas de alto nivel si una proposición los acompaña (¡Vaya, qué sorpresa!) o que activen descripciones cuando esa proposición no está inmediatamente al lado (Wharton, 2003). Por ejemplo, al contrario que en el caso anterior, no encontraríamos ipuf, qué cansancio!, sino que a partir de puf se podría rescatar esa noción (qué cansancio) sin tener que expresarla explícitamente gracias al contexto situacional o lingüístico.

Con la finalidad de llevar a cabo un estudio más pormenorizado de las CCEE, se presenta esta taxonomía para clasificar los distintos tipos de construcciones expresivas de alivio (Imagen 1) siguiendo como punto de partida la ofrecida por Igareda (2017: 34-347) ${ }^{3}$ :

1. CCEE producidas biológicamente por humanos, consideradas como CCEE vocálicas y/o consonánticas no léxicas ${ }^{4}$ porque no siguen los patrones léxicogramaticales de su lengua (estornudos, suspiros, risas...). Su máxima es la fidelidad fonética.

2. CCEE convencionalizadas: entendidas como elemento estándar y representativo en las diferentes lenguas. Su forma léxica suele representarse de la misma forma en diferentes idiomas, aunque a menudo su fonética aparece adaptada (como oh, uf).

3 Igareda distingue diez categorías de sonidos: (1) sonidos no producidos por humanos, (2) sonidos producidos biológicamente por humanos, (3) sonidos de animales, (4) de la naturaleza, (5) sonidos por la interacción humano-objeto, (6) pausas y sonidos de duda, (7) sonidos vocálicos no léxicos, (8) interjecciones convencionalizadas, (9) sonidos onomatopéyicos no fonológicos y (10) palabras ideofónicas.

4 Este tipo supone una mezcla entre las categorías de sonidos propuesta por Igareda (2017: 346): sonidos producidos biológicamente por humanos y sonidos vocálicos no léxicos. 
3. CCEE semánticas: construcciones cargadas de contenido semántico-emocional, pero sin una aparente realización fonético-prosódica específica (por ejemplo, gracias a Dios).

4. CCEE fonéticas: construcciones cuya entonación fonético-prosódica manifiesta un determinado contenido semántico-emocional, pero sin una forma léxica concreta (por ejemplo, Ssshah al aliviar una herida con agua).

5. CCEE ideofónicas ${ }^{5}$ : construcción de base icónica con un determinado contenido semántico-emocional y una realización fonético-prosódica específica (fiu para indicar alivio o shh para pedir silencio).

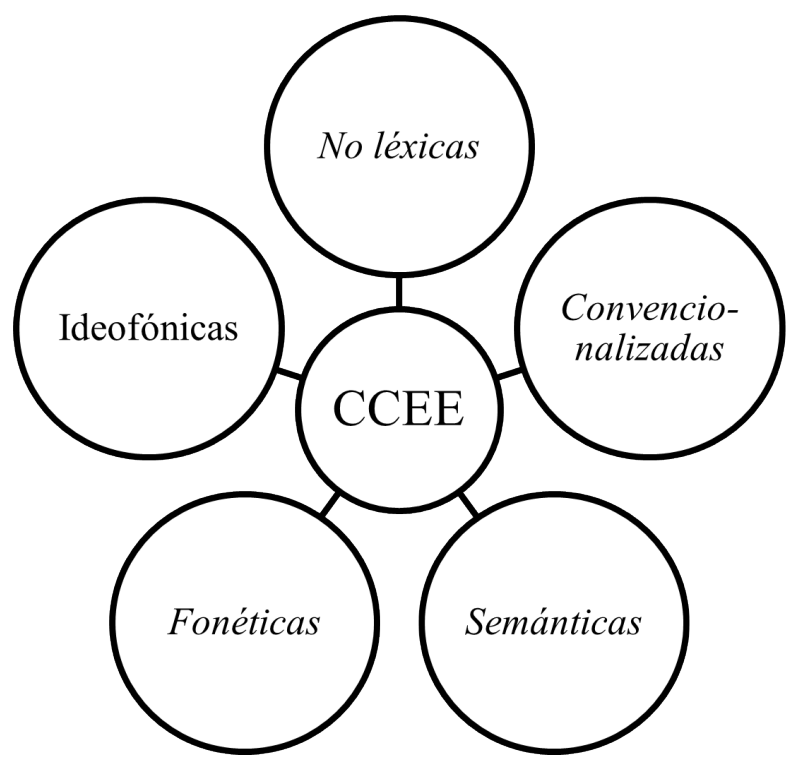

Imagen 1. Taxonomía de los tipos de CCEE.

Desde el punto de vista semántico, para la traducción de CCEE se debe tener en cuenta que una misma construcción puede albergar diferentes significados, según el contexto en el que se inserte. Así, la voz francesa oh, que es definida por el diccionario Larousse como "[interjection qui] sert à exprimer l'étonnement, l'admiration, la supplication, le dégoût, la répulsion, etc. ${ }^{\prime 6}$ muestra un abanico de significaciones emocionales diferentes. Según el tipo de emoción suscitada, se pueden apreciar tres tipos de CCEE a nivel semántico: i. aquellas con una significación emocional prototípica como fiu (español) y pfiou (francés) para expre-

5 "Los ideófonos [...] ejemplifican muy bien la iconicidad, es decir, la relación motivada entre la forma y el significado. Son estructuras que dadas sus características estructurales (fonético-prosódicas y morfo-sintácticas) [...] representan el significado de una manera muy vívida" (Ibarretxe-Antuñano, 2020: 412).

6 Traducción: “[interjección que] se utiliza para expresar asombro, admiración, súplica, asco, repulsión, etc.". 
sar alivio; ii. con varias significaciones emocionales especificadas por el contexto (polisémicas), como en el caso de la voz uh española, que denota desilusión o desdén $(D R A E)^{7}$; y iii. la misma concepción que la anterior, pero con significados emocionales opuestos, como el caso de $o h^{8}$, que expresa tanto admiración como repulsión dependiendo de su contexto y de la entonación con la que se pronuncie. Estas últimas construcciones las denominamos construcciones expresivas enantiosémicas, esto es, aquellas que revelan significados antónimos sin variar la forma de la construcción ( $c f$. Karaman, 2008; Odilov, 2016).

\subsection{Bilingüismo, emociones y multimodalidad en la traducción}

La naturaleza de las CCEE convierte en imprescindible la consideración de tres aspectos fundamentales durante el proceso de traducción: el bilingüismo, las emociones y la multimodalidad.

En primer lugar, en este estudio comparativo entre el inglés (lengua origen) y el francés (lengua meta), la supremacía de la lengua inglesa, considerada actualmente como la lengua vehicular a nivel internacional, reduce la reproducción de las CCEE a otras lenguas como ocurre con el francés y el español, por ejemplo. Algunos análisis de traducción entre el inglés y el francés evidencian una tendencia a mantener el inglés en el caso de las CCEE (Igareda, 2017; Richet, 2001), aunque para otros investigadores se trate de un "bilingüismo aditivo" y no "sustractivo" (Ben-Rafael, 2008).

En segundo lugar, los diversos significados emocionales que acompañan a las CCEE las convierten en un desafío en el proceso de traducción, puesto que precisan de técnicas creativas y de un conocimiento específico al no estar repartidas y codificadas de la misma manera a nivel sociocultural (Rojo López \& Ramos Caro, 2016; Sierra Soriano, 1999). Estas diferencias no tienen por qué radicar solo en la existencia o no de la emoción, sino que su forma de expresarla o entenderla a nivel cognitivo y paralingüístico también puede variar.

En tercer lugar, la característica multimodal de las emociones supone otro factor a tener en consideración durante la traducción. No solo es la forma en la que se pronuncie la construcción expresiva y el contexto que lleve asociado, sino que los gestos que la acompañan tanto faciales como corporales también son determinantes. Las emociones están conectadas con la prosodia y la entonación (Bänziger et al., 2001), pero principalmente con los movimientos corporales y faciales, siendo estos fundamentales en la comunicación multidimensional simultánea (Allwood, 2002; McNeill, 1992).

Dada la importancia de la parte verbal (fonética) y de la visual (paralingüística) de estas CCEE, los cómics se configuran como una de las plataformas más efectivas para llevar

7 REAL ACADEMIA ESPAÑOLA: Diccionario de la lengua española, $23^{\mathrm{a}}$ ed., [versión 23.4 en línea]. $<$ https:// dle.rae.es>.

8 En español, esta interjección se emplea "para manifestar muchos y muy diversos movimientos del ánimo, y más ordinariamente asombro, pena o alegría" (DRAE), observando esa significación opuesta entre pena y alegría. 
a cabo su análisis, debido a su intrínseca naturaleza multimodal (Borodo, 2014; Pratha et al., 2016). Tal y como señala Eisner: "the format of comics presents a montage of both word and image, and the reader [and the translator are] thus required to exercise both visual and verbal interpretative skills"9 (2008 [1985]: 2).

\subsection{La emoción de alivio}

La emoción de alivio, que es en torno a la que gira este estudio traductológico, requiere de esa premisa multimodal para su análisis por su funcionamiento verbal y paralingüístico. El alivio no fue incluido como una de las emociones básicas (asco, felicidad, ira, miedo, sorpresa y tristeza) por Ekman (1992), pionero en la codificación de gestos faciales. Pese a ello, es considerado como una emoción que forma parte de nuestro día a día (Sweeny \& Vohs, 2012) y sobre la que versan en la actualidad cada vez más estudios (Cordaro et al., 2017; Kamiloğlu, et al., 2020).

La emoción de alivio se caracteriza por su valencia positiva y una intensidad relativamente baja (Pekrun et al., 2011; Ganotice et al. 2016). Su intensidad es media-baja porque el alivio es el resultado de experimentar una emoción con valencia negativa que conduce a una positiva. Así, su valencia viene determinada por el estado final del sujeto experimentador, que es un estado emocional positivo.

La agrupación en diferentes familias que Sauter (2017) realiza de las emociones positivas permite encuadrar a la emoción con más precisión dentro de las que denomina como emociones positivas epistemológicas, esto es, aquellas emociones que suponen un cambio en la comprensión o el conocimiento que el individuo tiene sobre el mundo. Este cambio se produce debido a que una experiencia negativa ha cesado, se sabe que va a terminar en un futuro o porque un evento negativo ha sido menos malo de lo que se pensaba.

Una de las manifestaciones más prototípicas del alivio es el suspiro. Según la regulación de la dinámica de la respiración propuesta por Vlemincx y sus colaboradores (2017), desde un punto de vista fisiológico, el suspiro involucra un proceso de restauración del control respiratorio manteniendo un estado estable del sistema respiratorio homeostático, que permite responder de forma flexible a las demandas ambientales. Llevado a un punto de vista psicológico, la función homeostática del suspiro es parecida, ayudando al restablecimiento de la calma después de una experiencia de estrés o de una alta intensidad emocional induciendo a una sensación subjetiva de alivio.

En suma, la delimitación de la terminología en cuanto a estas CCEE y a las emociones que las acompañan supone un factor importante, así como el empleo de un corpus de índole multimodal para su estudio. Con el objetivo de analizar de forma pormenorizada la traduc-

9 Traducción: "el formato de los cómics presenta un montaje tanto de palabra como de imagen, por lo que se requiere que el lector [y, por ende, el traductor] ejercite[n] sus habilidades interpretativas tanto visuales como verbales". 
ción de las CCEE de alivio del inglés al francés, en este artículo se presenta: en primer lugar, el número de CCEE de alivio recabadas en los cómics; en segundo lugar, el mantenimiento o traducción de las CCEE en su paso al francés junto a una clasificación de las CCEE de alivio atendiendo a su naturaleza y estrategia de traducción; y, en tercer lugar, un análisis multimodal de algunas CCEE de alivio destacadas por su singular reproducción en la lengua meta.

\section{Materiales y métodos}

Para la elaboración del corpus se han seleccionado un total de cuatro series de cómics estadounidenses: Y: The Last Man (Vaughan \& Guerra, 2003-2008), Fashion Beast (Moore \& McLaren, 2012-2013), Nailbiter (Williamson \& Henderson, 2014-2017) y Providence (Moore \& Burrows, 2015-2017) con sus correspondientes traducciones al francés. En las cuatro series aparecen personajes con rasgos humanos y realistas que permiten realizar un estudio multimodal más preciso, ya que los gestos corporales y faciales están más pronunciados. La extensión de cada una de ellas se ha recogido en la Tabla 1:

\begin{tabular}{ccc}
\hline Cómic & Capítulos & Páginas \\
\hline Y: The Last Man & 60 & 1485 \\
Fashion Beast & 10 & 256 \\
Nailbiter & 30 & 760 \\
Providence & 12 & 499 \\
\hline & Total & 3000 \\
\hline
\end{tabular}

Tabla 1. Extensión de las series de cómics.

El análisis de las CCEE de alivio y su traducción se ha realizado partiendo desde diferentes niveles tales como la semántica, la ortotipografía, la pragmática, la fonética y la paralingüística. Este contraste lingüístico y multimodal implica una visión innovadora, puesto que se fundamenta en la parte verbal y visual del contenido, partes asentadas sobre la base sociocultural de la lengua y, por tanto, imprescindibles para el sostenimiento de la coherencia textual y visual en la traducción.

Para el estudio multimodal de los casos problemáticos de traducción de las CCEE de alivio se ha seguido el método de Cohn (2016). Este último propone tres categorías para el análisis de las interacciones multimodales (Imagen 2), entendiendo que ambas (la verbal y la visual) pueden tener gramática (Cohn, 2013), aunque no siempre aparece, a saber: a) categoría autónoma, donde solo hay una modalidad: solo se representa la verbal (una viñeta solo con texto) o solo la visual (imagen sin texto); b) dominante, en la que ambas modalidades están presentes, pero solo una utiliza gramática: por ejemplo, podría mostrarse en una secuencia visual una viñeta con un edificio del que de una ventana sale un bocadillo (hoy sí que 
te voy a ganar) por lo que solo habría gramática verbal, mientras que en otra viñeta donde aparezcan dos boxeadores y se representen los golpes (paf), solo habría gramática visual; y c) asertiva, donde ambas modalidades están presentes y ambas utilizan gramática.

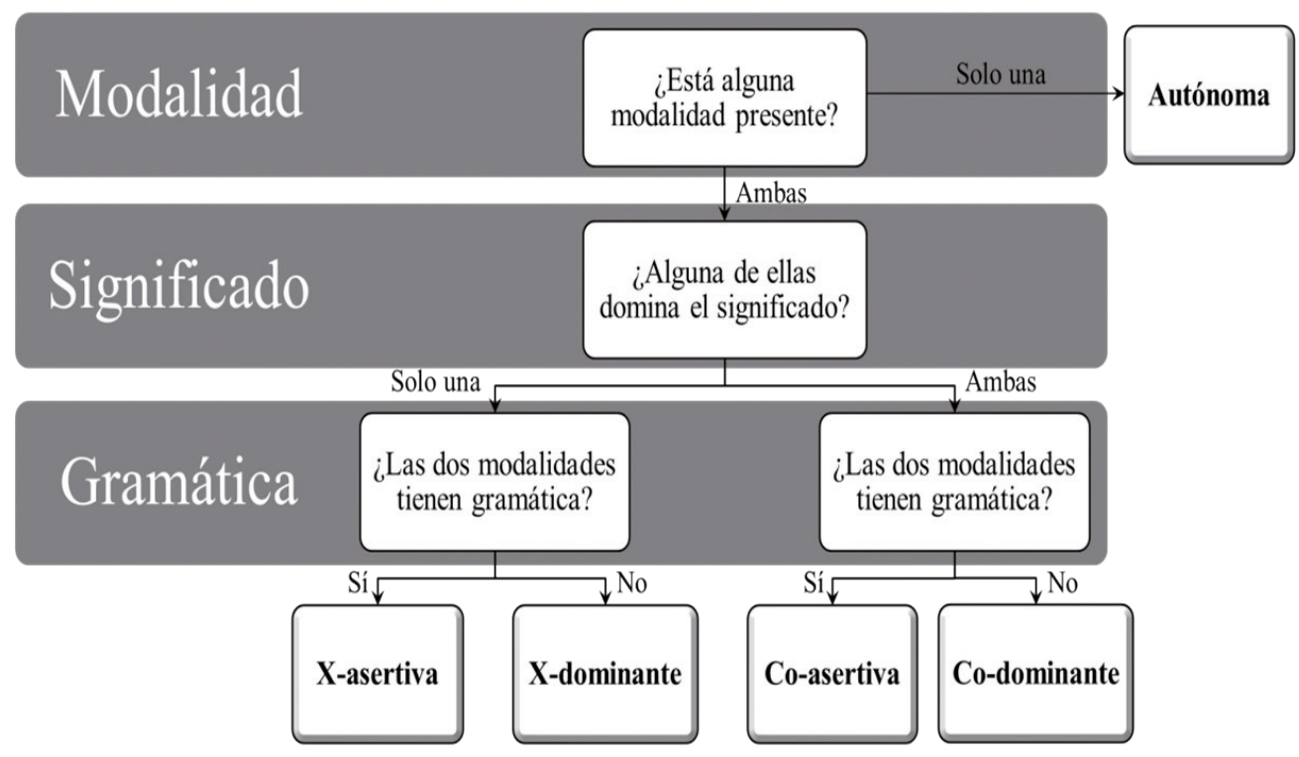

Imagen 2. Método paso a paso para el análisis de las interacciones multimodales (adaptado de Cohn, 2016: 30).

\section{Resultados y discusión}

\subsection{Número de CCEE de alivio}

La emoción de alivio está presente en el quehacer diario, es decir, en el desarrollo de las tareas y acciones de la vida (Vlemincx et al., 2017). Por esta razón, se contabiliza el número de CCEE de alivio de las cuatro series de cómics estadounidenses, que se recoge en la Tabla 2. Cabe mencionar que el total de $\mathrm{CCEE}^{10}$ está compuesto -siguiendo la terminología de Klett (2020)- por interjecciones, onomatopeyas y pseudogritos referidos a cualquier emoción, actitud o sonido ya sea su procedencia humana, animal o de objetos inanimados. El resultado es que el número de CCEE no referidas al alivio es mucho mayor que el de CCEE exclusivamente de esta emoción, suponiendo alrededor de un $1 \%$ del total.

10 No se han incluido en esta suma fórmulas de saludo y despedida ( hi, hello, howdy, bye, etc.), construcciones derivadas de adverbios de afirmación o negación (yeah, yep, etc.) o las utilizadas para apelar ya sean nombres comunes (man, boy, etc.) o verbos en imperativo (listen, look, see, etc.). 


\begin{tabular}{ccc}
\hline Cómic & CCEE & CCEE de alivio \\
\hline Y: The Last Man & 1.296 & 15 \\
Fashion Beast & 236 & 1 \\
Nailbiter & 734 & 4 \\
Providence & 929 & 5 \\
\hline Total & 3.195 & 25 \\
\hline
\end{tabular}

Tabla 2. Número total de CCEE de alivio en el corpus.

Pese a la consideración de la emoción de alivio como una emoción positiva que aparece de forma regular y cotidiana, el número de CCEE referidas a esta emoción es muy reducida tal y como se puede observar en la Tabla 2. Sin embargo, en la Tabla 1 se ha expuesto que el corpus utilizado para llevar a cabo este estudio está compuesto de tres mil páginas de las cuales se han extraído más de tres mil CCEE. Independientemente de su tipo, se esperaba que el número de CCEE de alivio fuera mayor. De esta forma, se dilucida que no es una emoción muy utilizada en los cómics en los que parece que se opta por otro tipo de emociones tales como la sorpresa o el miedo. Este hecho puede deberse a que estas últimas mantienen al lector más ensimismado en la lectura y reducen el empleo de CCEE de alivio para resoluciones finales de la acción donde se quiere remarcar ese fin de la emoción negativa, primando la positiva. Así, el contraste entre la aparición de emociones es uno de los puntos de investigación futura para observar qué relación existe entre la emoción de alivio y el resto de emociones, qué papel cumple dentro de la narrativa visual y por qué explicitarla en unas ocasiones y en otras no.

\subsection{Mantenimiento o traducción de las CCEE: tipos y estrategias de traducción}

En los estudios de traducción que versan sobre el paso de las CCEE de origen inglés a otras lenguas se manifiesta que su mantenimiento prevalece al trasladarlas a la lengua meta (Ben-Rafael, 2008). La Tabla 3 alberga la opción (la conservación o la modificación) por la que ha optado cada uno de los traductores de las cuatro series que constituyen el corpus de este trabajo. En este caso, se entiende como traducción cualquier cambio o adaptación que se haya realizado en el traslado de la construcción al francés, es decir, aquellas que reflejan alguna modificación con respecto a su forma original.

\begin{tabular}{lcccc|c}
\hline Cómic & Y: The Last Man & Fashion Beast & Nailbiter & Providence & \% \\
\hline CCEE de alivio & 15 & 1 & 4 & 5 & 100 \\
\hline Traducidas & 10 & 0 & 4 & 3 & 68 \\
Conservadas & 5 & 1 & 0 & 2 & 32 \\
\hline
\end{tabular}

Tabla 3. Traducción de CCEE de alivio. 
De este modo, se observa que la mayor parte de las CCEE de alivio son modificadas en su paso del inglés al francés, reflejando la importancia que tienen estas construcciones en los estudios de traducción. De ese $68 \%$ de construcciones de alivio traducidas la gran mayoría se corresponde con interjecciones, mientras que el 32\% de construcciones conservadas lo constituye principalmente onomatopeyas y pseudogritos.

En la Tabla 4 se exponen las estrategias de traducción más empleadas cuya clasificación se ha tomado de Molina y Hurtado (2002) y la clasificación de las diferentes CCEE, convirtiéndose ambos criterios en esenciales para comprender el porqué de la conservación o la modificación de estas en su traducción al francés. 
Anales de Filología Francesa, n. ${ }^{\circ}$ 29, 2021

FERNANDO CASANOVA MARTÍNEZ

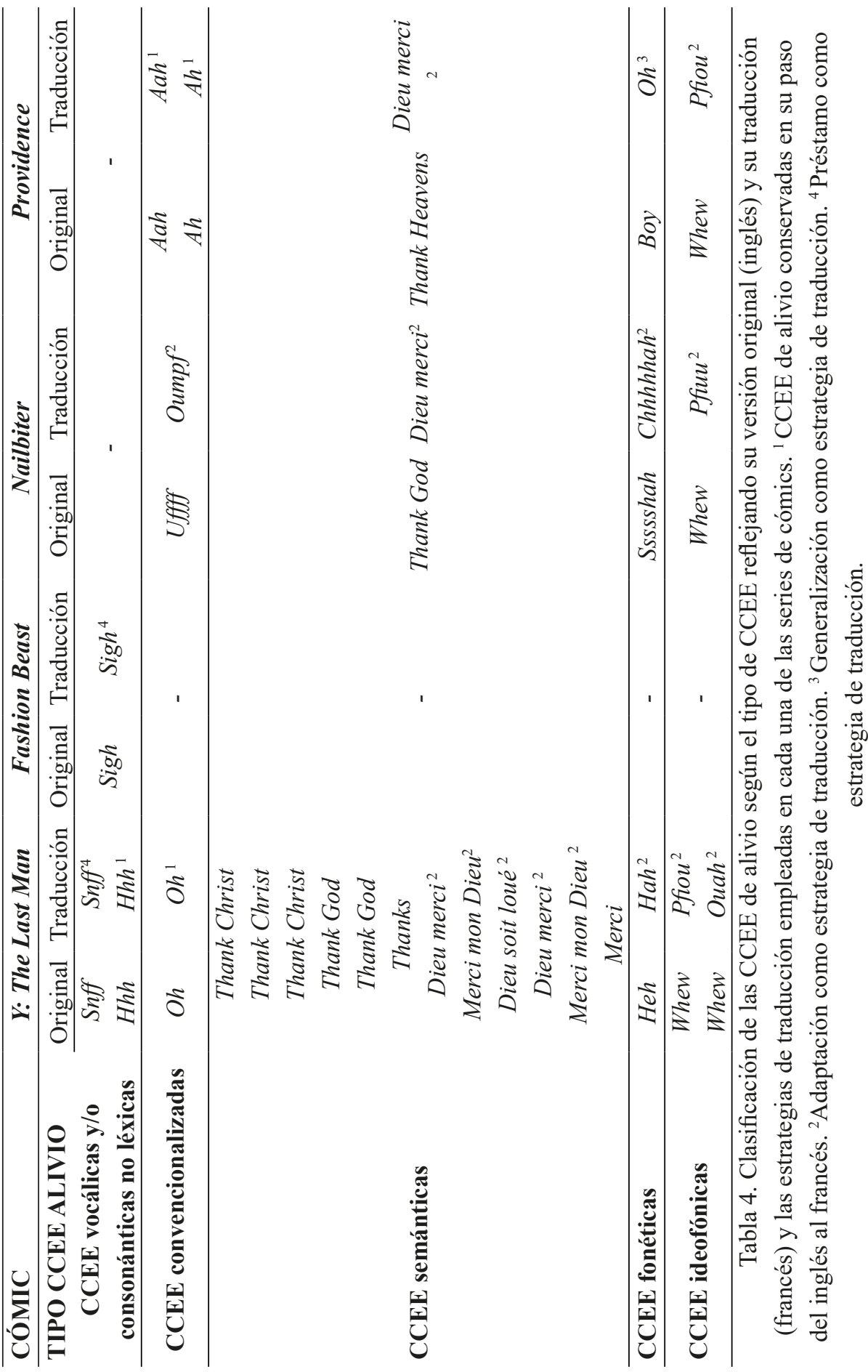


Respecto al factor de conservación o modificación de las CCEE de alivio del inglés al francés, se advierte que las CCEE vocálicas y/o consonánticas no léxicas y las CCEE convencionalizadas son las que no han experimentado ningún cambio en la traducción, salvo en el caso de Uffff $>$ Oumpf en el que se produce una adaptación ortotipográfica (analizada pormenorizadamente en el apartado 3.3.2.). El hecho de mantener intactas las CCEE del tipo $A h$ y $O h$ en el francés también es señalado por uno de los traductores participantes en el experimento traductológico de Richet (2001: 6), quien las denomina como "interjecciones vocálicas", pero asevera que el aspecto fonético y sociocultural de cada lengua debe ser tenido en cuenta. Por esta razón, puede darse una preferencia por el uso de una vocal u otra, apareciendo el fenómeno de la intercambiabilidad como sucede en la adaptación de las CCEE fonéticas Heh $>H a h$. Asimismo, los préstamos Snff (procedente del término inglés sniff 'oler') y Sigh ('suspirar') con una raíz inglesa evidente demuestran esa tendencia al mantenimiento del término inglés, sobre todo, en lo referente a sonidos (Igareda, 2017), ya que ni siquiera su fonética ha sido adaptada a la realización francesa habitual.

Por otro lado, las CCEE modificadas se corresponden con las CCEE fonéticas, semánticas e ideofónicas por su adherencia sociocultural ya sea a nivel fonético, semántico o ambos. En el caso de las CCEE semánticas, aunque pueda verse una conexión con la emoción positiva de gratitud desde la construcción Thanks $>$ Merci hasta las combinadas con un elemento religioso (Thank God>Dieu merci), presentan una connotación de alivio en el contexto en el que se insertan. Concretamente, en el caso de Thanks $>$ Merci el personaje agradece que haya llegado la hora de su muerte por su deseo de reunirse con un ser querido que podríamos equipararlo con la forma Sigh, que aparece en Fashion Beast, dado que ambos comparten el mismo deseo y son conocedores de su inminente cumplimiento. Así, la forma léxica de las CCEE tanto por razones fonéticas (Ssssshah>Chhhhhah, por ejemplo) como por razones semánticas (Thank Christ>Dieu soit loué) es trasladada para su adecuada comprensión y pronunciación en la lengua meta. Finalmente, en lo que respecta a las CCEE ideofónicas (Whew>Pfiou), manifiestan la base icónica de la respiración al producir alivio llegue a completarse como un suspiro o realizando una respiración específica (Schröder, 2003). Por tanto, cobra fuerza la idea planteada en Ben-Rafael (2008) quien considera que la traducción de las construcciones inglesas al francés no debe ser entendida como un bilingüismo invasivo, sino que más bien aditivo, puesto que no todas se mantienen.

\subsection{Análisis multimodal de las CCEE de alivio}

La multimodalidad es una característica inherente tanto de las CCEE como de los cómics y las emociones debido a su naturaleza paralingüística (visual) y fonética (verbal) (Chu \& Coffey, 2015; Dunst et al., 2018). De este modo, los cómics se erigen como herramientas fundamentales para el estudio de las CCEE conducido por métodos de corte multimodal, 
cognitivo y de corpus. En consecuencia, se realiza un análisis multimodal de cuatro CCEE que aparecen en los cómics estudiados con el fin de verificar su eficacia en la lengua meta. Para ello, se toma como punto de partida la presencia de gramática propia por parte de la imagen visual, a la manera de la sintaxis para el relato verbal, propuesta y desarrollada en la actualidad por investigadores como Cohn (2020) y colaboradores de esta tendencia teórica (Cohn \& Magliano, 2020). Con este fin, se sigue el esquema del método para el análisis de las interacciones multimodales anteriormente citado en la Imagen 2 (Cohn, 2016).

\subsubsection{Snff $>$ Snff}

La conservación de esta construcción consonántica no léxica en el francés refleja la influencia anteriormente comentada que ejerce el inglés sobre esta lengua romance. Snff, que aparece en Y: The Last Man, procede del verbo to sniff el cual tiene dos significados: i. 'oler' que aparece representado en este mismo cómic con la construcción snf (de nuevo expresada sin cambios tanto en el original como en la traducción) acompañada de la proposición "Is that perfume?” (¿Es un perfume?); y ii. 'aspirar’ o 'inspirar’ que parece la significación más apropiada para este ejemplo, ya que el personaje se sienta en su sillón mirando la foto de su marido fallecido tras unos días de mucho ajetreo acostumbrada a estar sola y serena (Imagen 3). De esta manera, el personaje vuelve a recuperar la tranquilidad de su hogar y se siente aliviada. A una inspiración le sigue una espiración por lo que esta construcción puede marcar un patrón respiratorio parecido al suspiro, que es la muestra de alivio por excelencia (Vlemincx et al., 2017), aunque solo se representa la primera parte, es decir, la inspiración. Una de las formas que podría proponerse para la traducción de esta construcción, así como para Sigh, que aparece también conservada en Fashion Beast, sería Soupir (suspiro en francés). Así, se optaría por un término propio de la lengua meta y no por el uso de un préstamo del inglés asegurándose el correcto entendimiento del significado emocional.

El análisis multimodal de esta construcción expresiva de alivio sería el siguiente: i. se constata que ambas modalidades (la verbal y la visual) están presentes; ii. el significado recae sobre ambas modalidades, apoyado por el contexto que envuelve a la secuencia: la visual permite observar que el personaje está tocando con la mano la foto de ella y de su marido recostada en el sillón, expresando nostalgia, calma o alivio al volver a estar con él. No obstante, no se puede saber con exactitud qué está sintiendo debido a la ausencia de gestos faciales. Por esta razón, la construcción verbal también es imprescindible porque aporta el matiz semántico que ayuda a discernir entre las emociones anteriores al representarse la parte inicial del patrón respiratorio de un suspiro; y iii. la única modalidad que tiene gramática es la visual, puesto que se considera que las CCEE que no siguen las normas sintáctico-gramaticales no tienen gramática. Por tanto, esta secuencia se clasifica como una co-dominante en la que sobre ambas modalidades recae el peso del significado, pero solo una tiene gramática. 


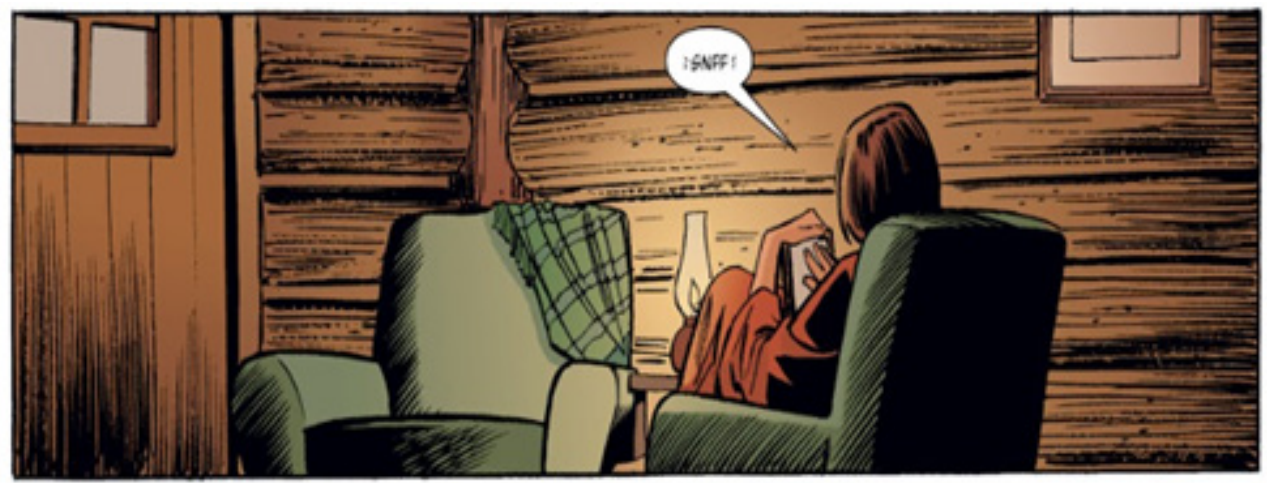

Imagen 3. Mantenimiento de la construcción 'Snff" inglesa en su paso a la lengua francesa (cómic: Y: The Last Man).

\subsubsection{Uffff $>$ Oumpf}

En el caso de la construcción expresiva convencionalizada Uffff $>$ Oumpf se produce una adaptación fonética en su paso al francés dando lugar a una reducción del énfasis fonético. No obstante, parece que más bien se origina un cambio en el modo de articulación: de una cadena de cuatro fricativas labiodentales, que indican la salida del aire por la boca, a una nasal seguida de una realización africada (oclusiva + fricativa). De esta manera, se observa que, según el idioma, el aire sale por diferentes vías aéreas: únicamente por la boca (inglés) y por la nariz primero y después por la boca (francés). De hecho, esta noción ya aparece representada en la parte visual con la boca semiabierta de la protagonista y la forma volátil del bocadillo (Imagen 4).

Teniendo en cuenta estos parámetros, esta unidad multimodal se analiza de la siguiente manera: i. ambas modalidades están presentes; ii. la predominancia del significado se ajusta a la parte visual de la viñeta, puesto que, aunque no estuviera manifestada la construcción, gracias a los gestos faciales se entendería perfectamente que la protagonista acaba de suspirar al acostarse en la cama después de un día duro de trabajo. En este caso, la forma del bocadillo también ayuda a comprender esa salida de aire que acompaña a la construcción; y iii. solo la parte visual goza de gramática, dado que se considera que las CCEE que no siguen las normas sintáctico-gramaticales no tienen gramática. Por tanto, se trata de una dominante visual, clasificación que encuadra con los datos aportados anteriormente y por su estatus de CCEE convencionalizada como elemento estándar y conocido en diferentes lenguas. 

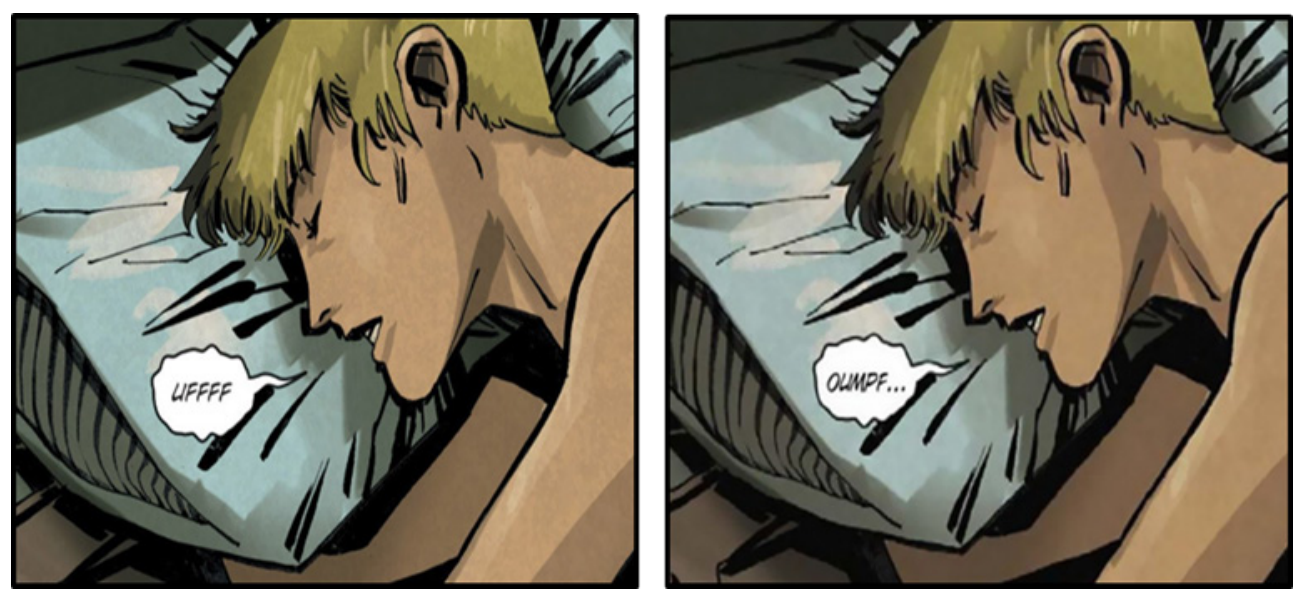

Imagen 4. A la izquierda la construcción "Uffff” de la versión original inglesa y a la derecha su traducción francesa “Oumpf” (cómic: Nailbiter).

\subsubsection{Boy $>\mathrm{Oh}$}

Esta construcción expresiva fonética, que aparece en el cómic Providence, resulta de especial interés debido al cambio que se ha producido en su paso del inglés al francés mediante el uso de una generalización. De esta manera, en la versión original inglesa se había optado por una construcción que, lejos de ser una apelación, revela un significado emocional de alivio, que incluso se explicita a su lado en el bocadillo "Boy, is that a relief!" o en su traducción al francés (“Oh, quel soulagement!”) (Imagen 5). Precisamente esta es la clave para entender que ese "boy” no es una apelación sin más, sino que está pronunciado de una forma específica que expresa esa emoción de alivio.

Atendiendo al método de análisis multimodal se observa que: i. tanto la modalidad verbal como la visual están presentes; ii. la parte verbal domina el significado, puesto que si solo tuviéramos la visual, se observaría que el personaje está movido por una emoción positiva, pero no se podría saber con certeza cuál hasta que se leyera el texto; y iii. ambas modalidades tienen gramática. Por ende, esta unidad multimodal se configura como una asertiva verbal $^{11}$, esto es, aquella en la que predomina el significado verbal estando presentes ambas modalidades y disponiendo ambas de gramática, por lo que queda patente esa importancia de la parte verbal (fonética) de la construcción clasificada como una construcción expresiva fonética.

11 Este análisis sería similar para las construcciones $H e h>$ Hah y Whew $>$ Ouah. 

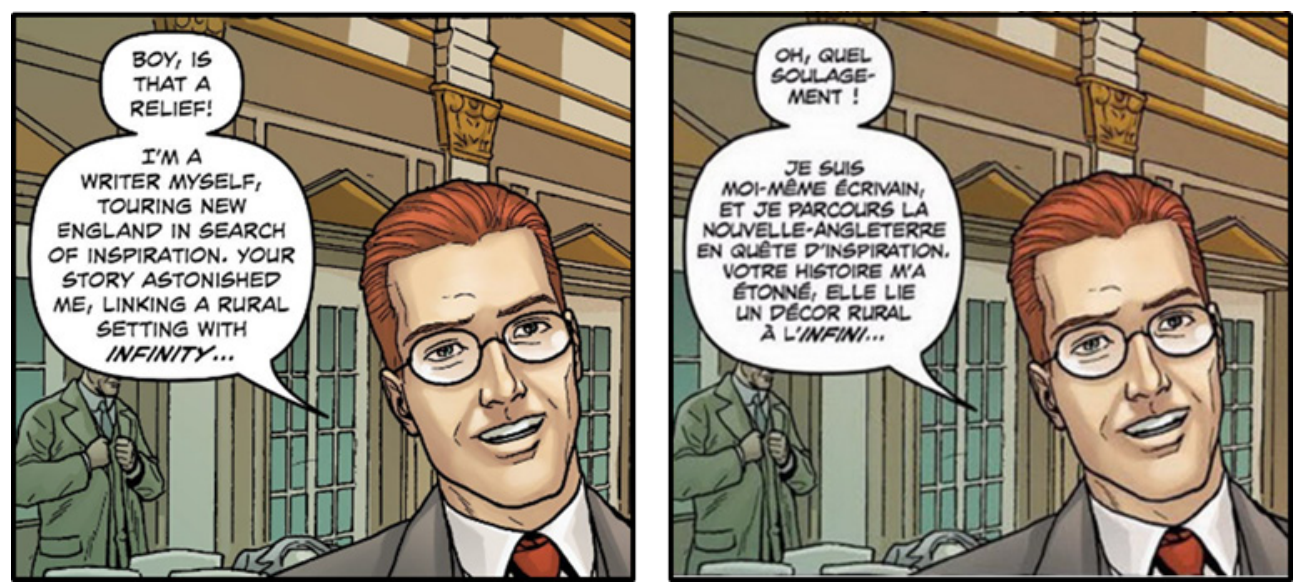

Imagen 5. A la izquierda la construcción "Boy" de la versión original inglesa y a la derecha su traducción francesa “Oh" (cómic: Providence).

\subsubsection{Whew $>$ Ouah}

La traducción de esta construcción expresiva ideofónica del inglés (Whew) al francés (Ouah) en Y: The Last Man ha experimentado cambios importantes. La opción escogida para el francés se utiliza para manifestar 'afirmación' (como una manera informal de oui) o 'sorpresa'. Por tanto, será el contexto lingüístico/situacional o los gestos los que nos permitan desambiguar y entender si es una situación de alivio o no, ya que las significaciones emocionales prototípicas del francés no apostarían por esta emoción. El contexto de esta secuencia visual es el siguiente: una chica encuentra a su pareja hablando aparentemente sola por lo que experimenta una emoción negativa porque, debido a problemas del pasado, sufre algunos desvaríos. La chica le pregunta si estaba hablando sola a la otra y esta última le responde negativamente, asegurándole que estaba cantando. El contenido verbal que sigue a la construcción en cada caso es: en la versión original ("Whew, excited about dinner?") y en la traducción (“Ouah. C'est le dîner qui t'enthousiasme?”). Ante esta situación la versión original opta por manifestar alivio "Whew" por el hecho de que ella no esté hablando sola, mientras que la versión traducida indica sorpresa "Ouah", conectando la construcción con el contexto posterior (positivo) y no el anterior (negativo), es decir, manifestando sorpresa por las ganas de cenar que tiene la chica. De este modo, las construcciones en una lengua y otra se refieren a hechos distintos y esta es la razón por la que son tan distintas. Con la pretensión de mantener el significado emocional de alivio del original, al utilizar esta adaptación se debería haber seleccionado la construcción ideofónica Pfiou como sí que ha ocurrido en otro momento en el mismo cómic.

Como se ha indicado en el ejemplo anterior, en el análisis se observa que esta unidad multimodal también se clasifica como una asertiva verbal porque los gestos faciales no nos 
permiten discernir qué emoción positiva se está transmitiendo sin acudir al contenido verbal adyacente por lo que el significado predominante es el verbal (Imagen 6).
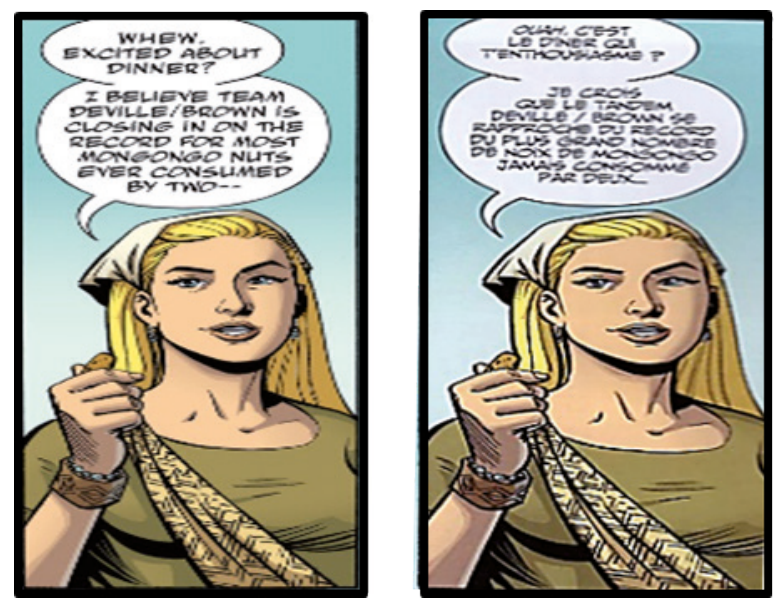

Imagen 6. A la izquierda la construcción "Whew" de la versión original inglesa y a la derecha su traducción francesa "Ouah" (cómic: Y: The Last Man).

\section{Conclusiones}

Tras este recorrido por las traducciones de las CCEE de alivio del inglés al francés en cuatro series de cómics estadounidenses diferentes, se constata que, a pesar de ser una emoción que se experimenta de forma rutinaria en el día a día, no presenta unos altos índices de aparición en los cómics. Una posible falta de representación de esta emoción puede deberse a la enfatización de otras que mantienen sediento al lector como la sorpresa o el miedo, mientras que la emoción de alivio es una emoción más próxima al resultado de la acción y no a su desarrollo.

Por su parte, la correcta selección de la terminología en lo que se refiere al estudio de las CCEE se convierte en un aspecto esencial para el correcto entendimiento del estudio que se ha realizado. Para ello, se ha empleado el término amplio de construcciones expresivas para englobar a interjecciones, onomatopeyas y sonidos inarticulados, concretándose, más tarde, los diferentes tipos de CCEE (vocálicas y/o consonánticas no léxicas, convencionalizadas, semánticas, fonéticas e ideofónicas).

Asimismo, resulta imprescindible que más estudios versen sobre la traducción y funcionamiento de estas CCEE debido a que la mayoría son traducidas en su paso a la lengua meta, pese a las tendencias de mantenimiento de la versión inglesa por la supremacía de esta lengua. En línea con lo anterior, las estrategias de traducción que destacan para la traducción de CCEE de alivio son la adaptación y el préstamo. 
La propuesta innovadora de clasificación de las CCEE se aúna con el método de análisis multimodal, manifestando que la naturaleza de las CCEE tiene su correlato con el funcionamiento multimodal de la unidad. De esta manera, en la actualidad estamos llevando a cabo estudios sobre el factor cognitivo y multimodal de estas construcciones, especialmente sobre las CCEE semánticas, con el objetivo de constatar esa aparente falta de especialización fonético-prosódica. Esta y otras muchas aportaciones pueden contribuir a la mejora en los procesos de traducción, así como a arrojar más luz sobre las características y el funcionamiento de estas construcciones.

\section{Referencias bibliográficas}

Allwood, Jens. 2002. "Bodily Communication Dimensions of Expression and Content" in Multimodality in Language and Speech Systems, 7-26.

Almela PÉrez, Ramón. 1982. Apuntes gramaticales sobre la interjección. Murcia, Publicaciones de la Universidad de Murcia.

Alonso Cortés, Ángel. 1999. "Las construcciones exclamativas. La interjección y las expresiones vocativas" in Bosque, Ignacio \& Violeta Demonte (dirs.). Gramática descriptiva de la lengua española. Madrid, Espasa-Calpe, 3993-4050.

Bänziger, Tanja, Didier Grandjean, Pierre-Jean Bernard, Gudrun Klasmeyer \& Klaus Scherer. 2001. "Prosodie de l'émotion: étude de l'encodage et du décodage" in Cahiers de linguistique française, $\mathrm{n}^{\circ} 23,11-37$.

Ben-Rafael, Miriam. 2008. "English in French comics” in World Englishes, n²7, 535-548. Borodo, Michał. 2014. "Multimodality, translation and comics" in Perspectives: Studies in Translatology, n²3, 22-41.

Bottineau, Didier. 2013/4. “Oups! Les émotimots, les petits mots des émotions: des acteurs majeurs de la cognition verbale interactive" in Langue française, $\mathrm{n}^{\circ} 180,99-112$.

CHu, Kin-wai \& Simon Coffey. 2015. "Multimodal analysis of graphic novels: a case study featuring two Asian women travellers" in Intercultural Communication Studies, n²4, 145 166.

Conn, Neil. 2013. The visual language of comics: Introduction to the structure and cognition of sequential images. Londres, Bloomsbury.

CoHn, Neil. 2016. "A multimodal parallel architecture: A cognitive framework for multimodal interactions" in Cognition, nº146, 304-323.

CoHn, Neil. 2020. Who Understands Comics?: Questioning the Universality of Visual Language Comprehension. Londres, Bloomsbury.

Cohn, Neil \& Joseph Magliano. 2020. “Editors' Introduction and Review: Visual Narrative Research: An Emerging Field in Cognitive Science" in Topics in Cognitive Science, n¹2, 197-223. 
Cordaro, Daniel T, Rui Sun, Dacher Keltner, Shanmukh Kamble, Niranjan Huddar \& Galen MCNEIL. 2017. "Universals and Cultural Variations in 22 Emotional Expressions Across Five Cultures" in Emotion, 1-19.

CuencA, Ma Josep. 2000. "Defining the indefinable? Interjections" in Syntaxis, n³, 29-44.

Dunst, Alexander, Jochen Laubrock \& Janina Wildfeuer. 2018. Empirical Comics Research. Digital, Multimodal, and Cognitive Methods. Nueva York, Routledge.

EISNer, Will. 2008 [1985]. Comics and Sequential Art. Nueva York-Londres, W.W. Norton \& Company.

Ekman, Paul. 1992. "An argument for basic emotions" in Cognition and Emotion, nº, 169200.

Ellis, Nick, Ute Römer \& Matthew Brook O’Donnell. 2016. Usage-based Approaches to Language Acquisition and Processing: Cognitive and Corpus Investigations of Construction Grammar. Chichester, Wiley-Blackwell.

Ganotice, Fraide, Jesús Alfonso Datu \& Ronnel King. 2016. "Which emotional profiles exhibit the best learning outcomes? A person-centered analysis of students' academic emotions" in School Psychology International, n³7, 498-518.

Goddard, Cliff. 2013. 'Interjections and Emotion (with Special Reference to 'Surprise' and 'Disgust')"' in Emotion Review, n6, 53-63.

Goldberg, Adele. 1995. Constructions: A construction Grammar Approach to Argument Structure. Chicago, University of Chicago Press.

IbarretXe-Antuñano, Iraide. 2020. "Ideófonos y poesía” in Tropelias: Revista de teoría de la literatura y literatura comparada, $\mathrm{n}^{\circ} 7,411-425$.

IGAREDA, Paula. 2017. "Approach to the translation of sound in comic books" in Babel, n'63, 343-363.

KamiloĞLu, Roza, Agneta Fischer \& Disa Sauter. 2020. "Good vibrations: A review of vocal expressions of positive emotions" in Psychonomic Bulletin \& Review.

Karaman, Burcu Ilkay. 2008. “On contronymy” in International Journal of Lexicography, n²1, 173-192.

KLETT, Estela. 2020. Études contrastives français-espagnol: les onomatopées et les doublets sémantiques. Buenos Aires, Editores Asociados.

LAROusse: Dictionnaire Larousse en ligne: <https://www.larousse.fr/ > [10/05/2021].

LóPez Boвo, M Mesús. 2002. "Hacia una caracterización semántico-pragmática de la interjección” in Pragmalingüística, nº10-11, 177-202.

McNeILl, David. 1992. Hand and Mind: What Gestures Reveal about Thought. Chicago y Londres, University of Chicago Press.

Molina, Lucía \& Amparo HurTado. 2002. "Translation Techniques Revisited: A Dynamic and Functionalist Approach" in Meta: Journal Des Traducteurs, n47, 498-512. 
Anales de Filología Francesa, n. ${ }^{\circ}$ 29, 2021

ESTUDIO CONTRASTIVO Y MULTIMODAL INGLÉS-FRANCÉS DE LA TRADUCCIÓN DE CONSTRUCCIONES...

Moore, Alan \& Jacen Burrows. 2015-2017. Providence. Rantoul, Avatar Press.

Moore, Alan \& Jacen Burrows. 2016-2017. Providence. Niza, Avatar Press/Panini France.

Moore, Alan \& Malcolm McLaren. 2013. Fashion Beast. Laurent-du-Var, Panini France.

Moore, Alan \& Malcolm McLaren. 2012-2013. Fashion Beast. Rantoul, Avatar Press.

ODILOv, Yorqinjon. 2016. "Theoretical basis of enantiosemy and its occurrence in speech" in Sendebar, nº5, 102-108.

Pekrun, Reinhard, Thomas Goetz, Anne Frenzel, Petra Barchfeld \& Raymond Perry. 2011. "Measuring emotions in students' learning and performance: The Achievement Emotions Questionnaire (AEQ)" in Contemporary Educational Psychology, n³6, 36-48.

Pratha, Nimish, Natalie Avunjian \& Neil Cohn. 2016. "Pow, Punch, Pika, and Chu: The Structure of Sound Effects in Genres of American Comics and Japanese Manga" in Multimodal Communication, $\mathrm{n}^{\circ} 5,102-108$.

Real Academia Española. Diccionario de la lengua española [DRAE], 23. ${ }^{a}$ ed., [versión 23.4 en línea]: <https://dle.rae.es $>$ [10/05/2021].

Richet, Bertrand. 2001. "Quelques données et réflexions sur la traduction des interjections" in Ballard, Michel (éd.). Oralité et traduction. Arras, Artois Presses Université, 79-128.

Rojo López, Ana María \& Marina Ramos Caro. 2016. "Can emotion stir translation skill? Defining the impact of positive and negative emotions on translation performance" in $\mathrm{Mu}-$ ñoz Martín, Ricardo (ed.). Reembedding Translation Process Research. Ámsterdam, John Benjamins, 107-130.

SAuter, Disa. 2017. "The Nonverbal Communication of Positive Emotions: An Emotion Family Approach” in Emotion Review, n 9, 222-234.

SCHRÖDER, Marc. 2003. "Experimental study of affect bursts" in Emotion Review, n40, 99116.

SIERra SorIAno, Ascensión. 1999. "L'interjection dans la BD: réflexions sur sa traduction" in Meta: Journal Des Traducteurs, n 44, 582-603.

SweEny, Kate \& Kathleen Vohs. 2012. “On Near Misses and Completed Tasks” in Psychological Science, n²3, 464-468.

Vaughan, Brian \& Pia Guerra. 2004. Y: Le Dernier Homme. Paris, Semic, vol. 1-2.

Vaughan, Brian \& Pia Guerra. 2006-2010. Y: Le Dernier Homme. Saint-Laurent-du-Var, Panini France, vol. 3-10.

Vaughan, Brian \& Pia Guerra. 2003-2008. Y: The Last Man. Nueva York, Vertigo, vol. $1-10$.

Vlemincx, Elke, Michel Meulders \& James Abelson. 2017. "Sigh rate during emotional transitions: More evidence for a sigh of relief" in Biological Psychology, n¹25, 163-172. 
Anales de Filología Francesa, n. ${ }^{\circ}$ 29, 2021

FERNANDO CASANOVA MARTÍNEZ

Wharton, Tim. 2003. "Interjections, language, and the 'showing/saying' continuum" in Pragmatics \& Cognition, $\mathrm{n}^{\circ} 11,39-91$.

Williamson, Joshua \& Mike Henderson. 2016-2017. Nailbiter. Grenoble, Glénat, vol. 1-6.

Williamson, Joshua \& Mike Henderson. 2014-2017. Nailbiter. Portland, Image Comics, vol. 1-6.

WiLkins, David. 1992. "Interjections as deictics" in Journal of Pragmatics, n¹8, 119-158. 
